\title{
Dynamics of Immiscible-Fluid Displacement in a Capillary Tube
}

\author{
Min-Yao Zhou ${ }^{(a)}$ and Ping Sheng \\ Exxon Research \& Engineering Co., Route 22 East, Clinton Township, Annandale, New Jersey 08801
}

(Received 10 July 1989)

\begin{abstract}
Through first-principles hydrodynamic calculations, the macroscopic behavior of the displacement of immiscible fluids in a capillary tube is linked to the microscopic parameters governing the dynamics of the moving contact line. Comparison with experimental data reveals that the contact line is dragged by a frictional force varying as $U^{x}$, where $U$ is the mean flow velocity and $x=\frac{1}{2}$. Consideration of capillary-wave excitation at the fluid-fluid interface as the source mechanism of this frictional force yields predictions in excellent agreement with experimental data.
\end{abstract}

PACS numbers: 47.55.Kf, 03.40.Gc, 68.10.-m, 68.45.-v

The displacement of one fluid by another immiscible fluid is a phenomenon whose rich ramifications have been the subject of continued theoretical and experimental studies for the past few decades. In Hele-Shaw cells or in porous media, this displacement process is shown to give rise to complex fingering patterns whose correlation with the system parameters is only recently begun to be understood.' Apart from the pattern formation, but intimately related to it at the fundamental level, is the problem of the moving contact line, ${ }^{2-7}$ defined as the intersection of the moving fluid-fluid interface with the solid wall. Here the application of the traditional nonslip boundary condition is shown to yield a nonintegrable stress singularity at any finite velocity, ${ }^{4}$ thus implying the breakdown of at least some elements of the traditional physical picture.

There have been two approaches to the resolution of this problem depending on the wetting property of the fluids. When one of the fluids completely wets the solid, it has been shown that there can be a thin precursor film that lines the solid wall. ${ }^{1}$ In this case the fluid-fluid interface only intersects the fluid film and therefore does not have a true contact line. On the other hand, when the fluids partially wet the solid it was proposed that there is a region of size $l_{s}$ around the contact line in which the nonslip boundary condition breaks down. ${ }^{2-6}$ This latter picture was recently supported by results of Monte Carlo simulations, ${ }^{8,9}$ which explicitly demonstrated the slipping of the moving contact line. Slipping thus removes the physical singularity but makes the resulting macroscopic behavior of immiscible-fluid displacement directly dependent on the microscopic parameters close to the contact line.

In this Letter, we present results of first-principles hydrodynamic calculations on immiscible-fluid displacement in the partially wetting case. Through comparison with experimental data, it is found that besides the effects of viscous stress near the contact line, there is a frictional force between the moving contact line and the solid wall that is proportional to $U^{x}$, where $U$ is the average flow velocity and $x=\frac{1}{2}$. We propose that the source of the new frictional force is due to the excitation of capillary waves at the fluid-fluid interface. Consideration of this mechanism yields quantitative agreement with experimental data.

Consider two immiscible fluids labeled 1 and 2 in a capillary tube of radius $R$, assumed to be small enough so that the gravity effects may be neglected. At rest, the interface between the two fluids forms a static contact angle $\theta_{0}$ with the wall as shown in Fig. 1 . As a result of the capillary pressure, it is necessary to have a counterbalancing pressure difference of $p_{0}=p_{1}-p_{2}=-2 \gamma$ $\times \cos \theta_{0} / R$ to maintain the static state. We will carry out our calculations in the comoving frame in which the interface is always stationary. Any pressure difference in excess of $p_{0}$ is counterbalanced by the viscous pull of the moving tube wall. In dimensionless form, the relevant hydrodynamic equations governing the steady motion of the two fluids are the time-independent Navier-Stokes equation and the incompressibility condition:

$$
\begin{aligned}
& -\nabla p+\mathrm{Ca} \nabla^{2} \mathbf{v}=(\mathrm{Ca})(\mathrm{Re}) \mathbf{v} \cdot \nabla \mathbf{v}, \\
& \nabla \cdot \mathbf{v}=0
\end{aligned}
$$

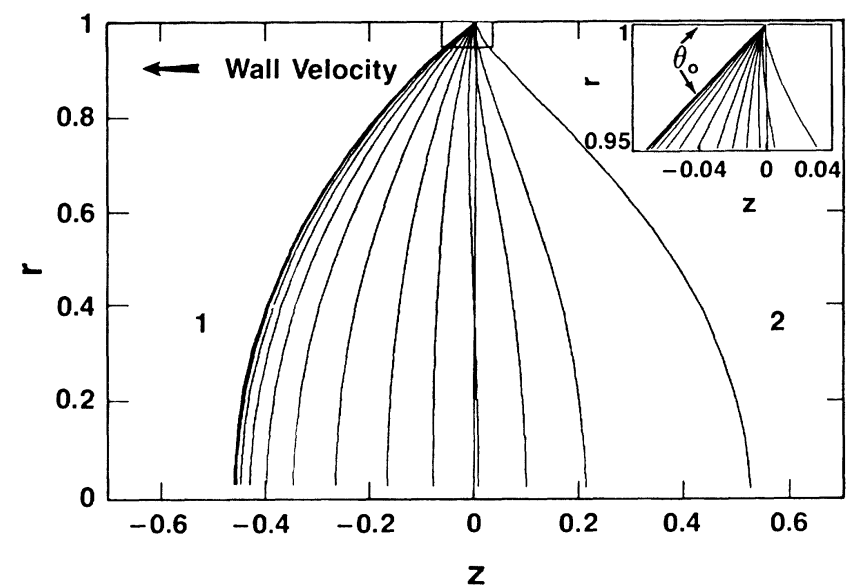

FIG. 1. Interfacial shape $Z_{0}(r)$ for different capillary numbers calculated with $\eta_{2} / \eta_{1}=1, l_{s}=10^{-4}$, and $\theta_{0}=40^{\circ}$ in model I. The leftmost profile is the static one. As $\mathrm{Ca}$ is increased from 0 to $10^{-4}, 2 \times 10^{-4}, 4 \times 10^{-4}, 8 \times 10^{-4}, 1.6 \times 10^{-3}$, $3.2 \times 10^{-3}, 6.4 \times 10^{-3}, 1.14 \times 10^{-2}, 1.64 \times 10^{-2}, 2.14 \times 10^{-2}$, $2.64 \times 10^{-2}, 3.14 \times 10^{-2}$, and $3.64 \times 10^{-2}$, the interface flips progressively to the right. Inset: An enlarged view of the contact-line region. The microscopic contact angle (clearly visible only within the slipping region) is fixed at $40^{\circ}$. 
where $p$ is the pressure field, $\mathbf{v}$ denotes the velocity field, $\mathrm{Ca}$ is the capillary number $\eta U / \gamma$, and $\mathrm{Re}$ is the Reynold's number $\rho U R / \eta$. Here $\eta$ and $\rho$ denote the viscosity and density of the two fluids in their respective domains, $\mathbf{v}$ is measured in units of $U, p$ is in units of $\gamma / R$, and length is in units of $R$. The nonlinear $\mathbf{v} \cdot \nabla \mathbf{v}$ term, while included in our calculations, shows negligible effect for all the cases addressed in this paper. Therefore $\mathrm{Ca}$ is the only controlling parameter in Eq. (1).

For the boundary conditions, we expect that due to viscous damping, far from the interface the flow should be Poiseuille-like. That means at $z= \pm L$, where we let $L=5, \partial v_{z} / \partial z=0, v_{r}=0$, and $p=$ const. At the fluid-fluid interface, we must have $\mathbf{v}_{1}=\mathbf{v}_{2}, \mathbf{v} \cdot \mathbf{n}=0$, where $n$ is the interface normal, and

$$
\left(p_{1}-p_{2}\right) \mathbf{n}=\left(\boldsymbol{\sigma}^{(1)}-\boldsymbol{\sigma}^{(2)}\right) \cdot \mathbf{n}+2 \gamma \kappa \mathbf{n} .
$$

Here $\sigma$ denotes the stress tensor of the fluid and $\kappa$ the principal curvature of the interface shape, $Z_{0}(r)$, which must also satisfy the requirements that at $r=1$ it forms an angle $\theta_{0}$ with the wall, and $d Z_{0}(r) / d r=0$ at $r=0$. For the fluid boundary conditions at the tube wall, $r=1$, we use two slipping models. In model I we let $v_{r}=0$ everywhere, $v_{z}=0$ at the slipping contact line, $z=0$ (since the contact line is defined to be stationary in our reference frame), and $v_{z}=-U\left[1-\exp \left(-|z| / l_{s}\right)\right]$ away from the contact line. In model II we adopt the Navier slipping model ${ }^{2}$ where the relative slipping velocity is related to the tangential stress at the wall, i.e., $v_{z}+U=\left(l_{s} / \eta\right) \sigma_{z r}$. In both cases $l_{s}$ is the physical parameter to be determined by comparison with experiments.

The numerical calculation was performed by first carrying out a hyperbolic-tangent-coordinate transformation such that the curved interface $Z_{0}(r)$ (parametrized), as well as the extra-fine spacing between the grid points around the contact line region $|1-r|$, $|z|<l_{s}$, is transformed to a regular Cartesian coordinate. The transformed Eqs. (1) and (2) are then solved by using the finite-difference method with the initial assumptions of a certain pressure difference and a spherical shape for $Z_{0}(r)$. We use Eq. (3) to match the $v$ and $p$ across the interface as well as to solve for $\kappa(r)$. From the solution a new $Z_{0}(r)$ is calculated by integrating the following differential equation: ${ }^{10}$

$$
\kappa(r)=\frac{1}{2} \frac{Z_{0}^{\prime \prime}+Z_{0}^{\prime}\left[1+\left(Z_{0}^{\prime}\right)^{2}\right] / r}{\left[1+\left(Z_{0}^{\prime}\right)^{2}\right]^{3 / 2}},
$$

with the initial conditions of $Z_{0}=0, Z_{0}^{\prime}=-\left(\tan \theta_{0}\right)^{-1}$ at $r=1$. Once $Z_{0}(r)$ is obtained, the slope $Z_{0}^{\prime}(0)$ is checked. If it is not zero, then the initially assumed pressure difference is modified and the calculation is iterated until consistency, in the sense that $Z_{0}^{\prime}(0)=0$ and the assumed $Z_{0}(r)$ equals the $Z_{0}(r)$ obtained, is achieved. Zero-sum checks, such as force balance and entropy generation minus the work done, show that our solution is accurate to $\sim 1 \%$ at $\mathrm{Ca}<10^{-3}$ and $\sim 5 \%-$
$10 \%$ at $\mathrm{Ca} \sim 10^{-2}$ provided that there are $5-10$ points inside the slipping region $|z|<l_{s}$.

Figure 1 shows the results of our calculated $Z_{0}(r)$ at various $C a$ values for model I with $l_{s}=10^{-4}$ and $\theta_{0}$ $=40^{\circ}$, assumed to be independent of velocity $\mathrm{Ca}$, and $\eta_{1}=\eta_{2}=\eta$. The interface is noted to flip from one side to the other as the Ca value is increased. This is true for both models I and II. The essential physics here may be stated as follows. At $\mathrm{Ca}=10^{-2}$, the viscous stress at the center of the tube is about 2 orders of magnitude smaller than the capillary pressure so that the interface would appear rigid to the fluid. However, near the contact line the near divergence of the viscous stress makes it comparable to the capillary pressure. The interface is therefore deformed by the viscous stress close to the contact line, making the "apparent" contact angle $\theta$ appreciably different from the actual contact angle $\theta_{0}$. Here $\theta$ is defined as $\theta=\tan ^{-1}\left[\left(1-h^{2}\right) / 2 h\right]$, where $h$ is the distance of the interface at $r=0$ from the $z=0$ plane. Experimentally, precisely this qualitative behavior has been observed as $U$ is increased, thus confirming one aspect of our results.

Our calculated results on the apparent contact angle for both models I and II also demonstrate the scaling behavior, ${ }^{3,6}$ which states that at a given value of $\mathrm{Ca}, \theta_{0}$ and $\theta$ are related by the equation

$$
g(\theta)=g\left(\theta_{0}\right)+\mathrm{Ca} \ln \left(K / l_{s}\right),
$$

where $K$ is a model-dependent constant, and the function $g(\theta)$ is given by Cox. ${ }^{6}$

Equation (5) implies that if $g$ has an inverse, then $\theta$ is a function only of the variable $y=g\left(\theta_{0}\right)+\mathrm{Ca} \ln \left(K / l_{s}\right)$, i.e., $\theta=g^{-1}(y)$. Therefore, two different $\theta_{0}$ values, $\theta_{0}^{(1)}$ and $\theta_{0}^{(2)}$, can yield the same apparent contact angle $\theta$ at values of $\mathrm{Ca}$ that differ by a constant amount of $\left[g\left(\theta_{0}^{(1)}\right)-g\left(\theta_{0}^{(2)}\right)\right] / \ln \left(K / l_{s}\right)$ so that they give the same $y$ value. It follows that the $\theta$ vs $\mathrm{Ca}$ curves calculated at different $\theta_{0}$ values may be superimposed upon each other through a uniform additive shift horizontally along the $\mathrm{Ca}$ axis. This is indeed verified. By fitting Eq. (5) to our calculated $\theta$ vs $C a$ results with fixed $\theta_{0}$, the ratio $K / l_{s}$ was obtained. Since $l_{s}$ is known (as input to the calculation), the value of $K$ may thus be extracted. We get $K=0.3$ for model $I$ and $K=0.05$ for model II. In fact, the different $K$ values represent the only macroscopic difference between the two models. The excellent agreement of Eq. (5) with our calculations means that Eq. (5), with the fixed $K$ value obtained above, may be used as a simpler way to evaluate the $\theta$ vs $\mathrm{Ca}$ behavior. Provided the behavior of $\theta_{0}$ is known, data fitting using Eq. (5) may yield the "experimental" values for $l_{s}$ within the framework of a given model.

The variation of the pressure difference with velocity is calculated to be $p_{1}-p_{2}=-2 \cos \theta+16(\mathrm{Ca}) L$. The second term is noted to arise from the viscous flow of a single fluid in a tube of length $2 L$. Since the pressure induced by the interface (the first term) can be expressed 
in terms of the apparent contact angle $\theta$, our results thus confirm the prediction of Dussan and Davis ${ }^{11}$ that $\theta$ essentially determines the macroscopic state of the immiscible flow. By holding everything equal and varying $l_{s}$, we find $\cos \theta \propto-\ln l_{s}$. This slow variation implies that for a given value of $\theta_{0}$, the $\mathrm{Ca}$ value at which $\theta$ reaches $180^{\circ}$, denoted as $(\mathrm{Ca})_{0}$, is very insensitive to $l_{s}$. The implication of this will be seen presently.

Experimental data on $\theta$ vs $\mathrm{Ca}$ have been obtained by Fermigier and Jenffer ${ }^{12}$ and Stokes et al. ${ }^{13}$ Some of their results are shown in Fig. 2. For given $\eta_{1}, \eta_{2}, U$, and $\gamma$, the inputs to our theory are $\theta_{0}$ and $l_{s}$. Since there are two parameters for every value of $\theta$, it may appear at first sight that there is no way to uniquely determine $\theta_{0}$ and $l_{s}$ from the data. However, we make the observation that physically, $l_{s}$ cannot be less than $1 \AA$. For $R \sim 1$ $\mathrm{mm}$, that means $l_{s} \gtrsim 10^{-7}$. Moreover, the insensitivity of $(\mathrm{Ca})_{0}$ to $l_{s}$ means that $l_{s}$ has to decrease by orders of magnitude before $(\mathrm{Ca})_{0}$ decreases by a factor of 2 . All this points to the fact that regardless of slipping models, if we let $\theta_{0}$ be velocity independent, then $\theta \approx \theta_{0}=$ const for $\mathrm{Ca}<10^{-2.7}$. In other words, any significant varia-

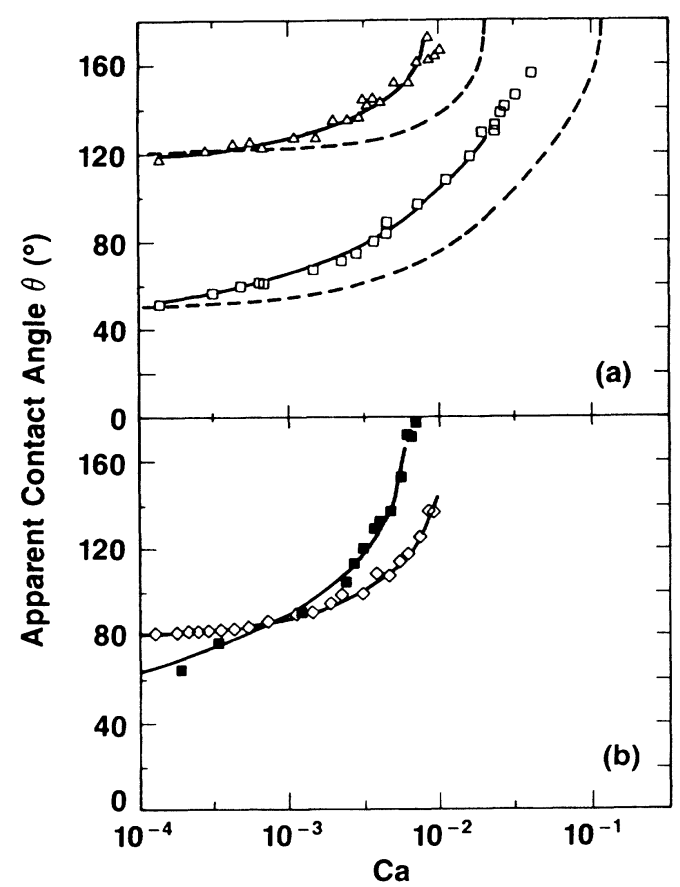

FIG. 2. Apparent contact angle plotted as a function of $\mathrm{Ca}$, defined here as $\eta_{1} U / \gamma$. (a) Data of Fermigier and Jenffer (Ref. 12). Triangles denote a system with $\eta_{2} / \eta_{1}=0.1$ and squares denote a system with $\eta_{2} / \eta_{1}=7.6 \times 10^{-3}$. The dashed lines are calculated from Eq. (5) with the appropriate $\eta_{2} / \eta_{1}$ ratios, $K=0.3$, and $l_{s}=10^{-7}$. Solid lines are calculated with $K=0.3, l_{s}=10^{-3}, B=5.4$, and $x=\frac{1}{2}$ for the triangle data and $K=0.3, l_{s}=10^{-5}, B=8$, and $x=\frac{1}{2}$ for the square data. (b) Data of Stokes et al. (Ref. 13). $\eta_{2} / \eta_{1}=1$ for both data sets. Solid lines are calculated from Eq. (5) with $K=0.3, l_{s}=10^{-5}$, $B=7.3, x=\frac{1}{3}$ for the filled-square data and $K=0.3, l_{s}=10^{-5}$, $B=5, x=\frac{1}{2}$ for the diamond data. tion of $\theta$ observed at $\mathrm{Ca}<10^{-2.7}$ can be attributed to the variation of $\theta_{0}$ away from its static value. From the force balance at the contact line, the variation of $\theta_{0}$ as a function of $U$ directly implies a frictional force per unit length $F$ given by

$$
F / \gamma=\cos \theta_{0}(0)-\cos \theta_{0}(U) \text {. }
$$

In Fig. 2(a) we show two sets of data of Fermigier and Jenffer ${ }^{12}$ measured in a tube with $R=0.5 \mathrm{~mm}$. The dashed lines are curves of $\theta$ vs $\log _{10}(\mathrm{Ca})$ calculated from Eq. (5) with $U$ independent $\theta_{0}, l_{s}=10^{-7}$, and $K=0.3$. They clearly disagree with the data. By analyzing the variation of $\theta$ for $\mathrm{Ca}<10^{-2.7}$, we found that $\theta_{0}(U)$ is related by Eq. (6) to a frictional force $F / \gamma$ that varies as $B(\mathrm{Ca})^{x}$ with $^{14} x=\frac{1}{2}$. The solid lines are the resulting fit to the data by using Eq. (5) in which the $U$ dependence of $\theta_{0}$ is given by Eq. (6), B and $l_{s}$ are the fitting parameters, and $\theta_{0}(0)$ is treated as a slightly adjustable initial value. As expected, the value of $B$ is most sensitive to the low-velocity data, whereas $l_{s}$ is determined by the higher-velocity data due to the dominance of the viscous effect at higher $U$, which also masks the effect of the friction force above $\mathrm{Ca}=10^{-2.7}$. Besides the excellent agreement obtained, the resulting values of $l_{s}$ are noted to be all within the physical range. In Fig. 2(b) we have another two data sets of Stokes et al. ${ }^{13}$ measured in tubes with $R=0.5$ and $1 \mathrm{~mm}$. The quality of the fits is equally good. However, now whereas in one case we still get the exponent value of $x=\frac{1}{2}$, in the other case the optimal exponent value is $x=\frac{1}{3}$.

Recent molecular-dynamic simulations have demonstrated $^{8,9}$ that for solid walls which are smooth on the molecular scale, $l_{s}=10 \AA$. However, Jansons ${ }^{15}$ has shown that for rough walls the "effective" slipping length that enters in the boundary condition to our hydrodynamic calculations should be on the order of the typical period of the random undulations, denoted as $l_{0}$. This effective slipping length arises from the jump of the contact line across indentations and therefore should be distinguished conceptually from the microscopic slipping length. Since roughness of the tube walls is expected to be present for the data shown in Fig. 2, our determination of the slip length, varying from $100 \AA$ to $1 \mu \mathrm{m}$ in different samples, is indicative of the roughness scale.

We propose that the same randomness-induced jerky motion of the contact line is responsible for the frictional force through the excitation of damped capillary waves at the fluid-fluid interface. The scale $l_{0}$ gives rise to a basic excitation frequency ${ }^{16} \omega_{0}=2 \pi U / l_{0}$ for the capillary wave. ${ }^{17}$ If the amplitude of our excitation is denoted by $\zeta=l_{0} g(w, t)$, where $w=1-r$ is the coordinate normal to the wall, then in accordance with the nature of the jerky motion $g(0, t)$ should be something like a sawtooth function where in one period there is a segment of slow motion with $\partial g(0, t) / \partial t \simeq U / l_{0}$ and another segment of fast motion, corresponding to the jump, ${ }^{18}$ in which the speed of the contact line $v$ is determined by 
the balance of the surface tension and viscous resistance, i.e., $v \simeq \gamma / \eta$. Since $U \eta / \gamma=\mathrm{Ca}$, we have $v / U=10^{3}$ if $\mathrm{Ca}=10^{-3}$. By decomposing $g(0, t)$ into a Fourier series in terms of the basis functions $\sin \left(n \omega_{0} t\right)$, we get

$$
g(w, t)=\sum_{n=1}^{\infty} C_{n} e^{-\alpha_{n} w} \cos \left(\beta_{n} w-\pi / 2+n \omega_{0} t\right),
$$

where $k_{n}=\beta_{n}+i \alpha_{n}$ is the wave vector corresponding to the frequency $n \omega_{0}$, and $C_{n}$ is the Fourier coefficient of the sawtooth function. Since the instantaneous reaction force on the wall is given by $f \simeq-\gamma \partial g(0, t) / \partial w$, the average rate of energy dissipation $F U$ may be equated to the time average of $f d \zeta / d t$ over one period. This yields $F / \gamma=\pi l_{0} \sum_{n} n C_{n}^{2} \beta_{n}$.

The dispersion relation for a damped capillary wave has been obtained by Huang and Webb: ${ }^{19}$

$$
S^{2}+\frac{1}{2} S\left[1+(1+2 S)^{1 / 2}\right]+y=0 \text {, }
$$

where $S=i \omega \tau_{0}, y=\Omega^{2} \tau_{0}^{2}, \tau_{0}=\rho / 2 \eta k^{2}, \Omega=\gamma k^{3} / 2 \rho, \rho$ being the average density, and $\eta=\eta_{1}=\eta_{2}$. Solution of Eq. (8) shows that for $\omega<\gamma^{2} \rho / 64 \eta^{3}=v_{0}$, there are two branches of solution for $k$, both of which give $\beta \propto \omega^{2 / 3}$. For $\omega>v_{0}$, however, we get $\beta \propto \omega^{x}$, where $x \approx 0.44$ at intermediate frequencies but approaches $x=0.5$ as $\omega \rightarrow \infty$, corresponding to the diffusive nature of the overdamped wave. For $\gamma=30 \mathrm{dyn} / \mathrm{cm}, \rho=1 \mathrm{~g} / \mathrm{cm}^{3}$, and $\eta=0.15 \mathrm{P}$, we get $v_{0}=5 \times 10^{3} \mathrm{sec}^{-1}$. Since the dissipation is dominated by the jump segment of the motion, the important frequency to be compared is $2 \pi v / l_{0}$ $=2 \pi \gamma / l_{0} \eta=10^{6}-10^{9} \mathrm{sec}^{-1}$ (for $l_{0}=10 \mu \mathrm{m}$ to $100 \AA$ ), which is much larger than $v_{0}$ so that one is always in the high-frequency regime. By taking the asymptotic solution of Eq. $(8), \beta_{n}=\left(n \omega_{0} \rho / 2 \eta\right)^{1 / 2}$, one gets

$$
\frac{F}{\gamma}=\left(\frac{\pi^{3} \gamma \rho l_{0}}{\eta^{2}}\right)^{1 / 2}\left(\sum_{n=1}^{\infty} C_{n}^{2} n^{3 / 2}\right)(\mathrm{Ca})^{1 / 2}=B(\mathrm{Ca})^{1 / 2} \text {. }
$$

From the values of $\gamma, \rho$, and $\eta$ given above, if one takes the experimental values of $l_{0}=10^{-6}-10^{-4} \mathrm{~cm}$ and $v / U=10^{-4}-10^{-2}$, we get $1<B<100$, which brackets the values of $B=5.4,5$, and 8 obtained from the data. Dissipation by capillary waves therefore provides not only the explanation for the exponent value of $x=\frac{1}{2}$, but also the agreement with the magnitude of the observed friction. The exponent value of $\frac{1}{3}$ is also within the estimated experimental error if we note that the two lowest-speed points have large uncertainties. In addition, recent accurate measurements ${ }^{13}$ focused on the lowspeed region have yielded an unambiguous value of $x=\frac{1}{2}$. We note that one testable consequence of our theory is that for molecularly smooth surfaces this velocity-dependent frictional force should be absent.

We wish to thank J. Stokes, M. Robbins, S. Bhattacharya, E. Charlaix, and A. Kushnick for many helpful conversations.

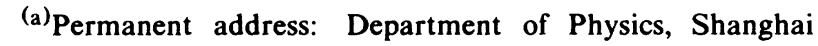
Teachers' University, Shanghai, People's Republic of China.

'See the review by P. G. de Gennes, Rev. Mod. Phys. 57, 827 (1985).

${ }^{2}$ See the review by E. B. Dussan, V, Annu. Rev. Fluid Mech. 11, 371 (1979).

${ }^{3}$ R. L. Hoffman, J. Colloid Interface Sci. 50, 228 (1975).

${ }^{4}$ L. M. Hocking, J. Fluid Mech. 76, 801 (1976); 79, 209 (1977).

${ }^{5}$ J. Lowndes, J. Fluid Mech. 101, 631 (1980).

${ }^{6}$ R. G. Cox, J. Fluid Mech. 168, 169 (1986).

${ }^{7}$ C. Huh and L. E. Scriven, J. Colloid Interface Sci. 25, 85 (1971).

${ }^{8}$ J. Koplik, J. R. Banavar, and J. F. Willemsen, Phys. Rev. Lett. 60, 1282 (1988).

${ }^{9}$ P. A. Thompson and M. O. Robbins, Phys. Rev. Lett. 63, 766 (1989).

10 J. J. Stoker, Differential Geometry (Wiley, New York, 1969), p. 131.

${ }^{11}$ E. B. Dussan, V, and S. H. Davis, J. Fluid Mech. 173, 115 (1986).

${ }^{12}$ G. M. Fermigier and P. Jenffer (to be published).

${ }^{13}$ J. P. Stokes, A. P. Kushnick, S. Bhattacharya, and M. Higgins (to be published).

${ }^{14}$ It should be noted that the earlier experiments by J. P. Stokes et al. [Phys. Rev. Lett. 57, 1718 (1986)] on the viscous fingering in porous media have suggested the existence of a $U^{1 / 2}$ type of capillary pressure. What we have found here could be its origin. Also, least-squares fitting of the data yields $x \approx 0.48-0.52$ for the best value in three cases and $x \approx 0.34$ in the other. However, the last data set does have large uncertainties for the two lowest-speed data points.

${ }^{15}$ K. M. Jansons, J. Fluid Mech. 154, 1 (1985); 167, 393 (1986).

${ }^{16}$ There is a close analogy between this oscillatory motion of the contact line and the motion of charge-density waves. See G. Grüner, A. Zawadowski, and P. M. Chaikin, Phys. Rev. Lett. 46, 511 (1981).

${ }^{17}$ Since the capillary wave is overdamped, the time-independent picture is still correct macroscopically provided we regard the effective slipping length and its associated $\cos \theta_{0}$ (and $F$ ) as quantities that are time averaged over the basic period $l_{0} / U$. Also, we have essentially neglected the lateral variation of the surface inhomogeneities around the tube. This would be correct if the damping length of the capillary wave, $\sim 1 \mu \mathrm{m}$, were less than the lateral correlation length of the inhomogeneities so each jump could be treated as independent. Otherwise, the lateral correlation may modify the value of $B$. The value of $x=\frac{1}{2}$ arises from the diffusive nature of the overdamped wave and is not expected to be modified.

${ }^{18}$ The analogous charge-density wave motion is discussed by D. S. Fisher, Phys. Rev. B 31, 1396 (1985). For $\mathrm{Ca} \simeq 1$, $U=\gamma / \eta$, and Reynold's number $=\gamma \rho l_{0} / \eta^{2}$ for jumps on the scale of $l_{0}$. For experimental values of $\gamma=30 \mathrm{dyn} / \mathrm{cm}, \eta=0.15$ $P, \rho=1 \mathrm{gm} / \mathrm{cm}^{3}$, and $l_{0}=1 \mu \mathrm{m}$, we get $\operatorname{Re} \lesssim 0.1$. That means the inertial effects may be neglected.

${ }^{19}$ J. S. Huang and W. W. Webb, Phys. Rev. Lett. 23, 1650 (1969). It should be noted that compared to energy loss due to the excitation of phonons and Stoneley waves, the capillarywave mechanism is favored due to its high density of states. 